\title{
Saponin Protects Against Cyclophosphamide- Induced Kidney and Liver Damage via Antioxidant and Anti-Inflammatory Actions
}

Mohammad Ghasem Golmohammadi

Ardabil University of Medical Sciences: Ardebil University of Medical Sciences

Shokofeh Banaei ( $\nabla$ s.banaei@arums.ac.ir)

Ardabil University of Medical Sciences https://orcid.org/0000-0002-8742-3895

Ali Abedi

Ardabil University of Medical Sciences: Ardebil University of Medical Sciences

\section{Research Article}

Keywords: Saponin, Cyclophosphamide, Liver, Kidney, Antioxidants

Posted Date: March 7th, 2022

DOl: https://doi.org/10.21203/rs.3.rs-1398111/v1

License: (c) (i) This work is licensed under a Creative Commons Attribution 4.0 International License.

Read Full License 


\section{Abstract \\ Purpose}

The liver and kidney are organs affected by chemotherapy drugs such as cyclophosphamide (CP). This study examined the protective effects of treatment with saponin (SP) against CP-induced nephrotoxicity and hepatotoxicity.

\section{Methods}

24 adult male mice were divided into four groups $(\mathrm{N}=6)$ : Control group, CP $(15 \mathrm{mg} / \mathrm{kg}), \mathrm{SP}(2.5 \mathrm{mg} / \mathrm{kg})$ and $\mathrm{CP}+\mathrm{SP}$. After treatment, the blood samples were collected for the determination of biochemical parameters and liver and kidney samples were taken for histological analysis and the assessment of oxidative stress and inflammatory markers.

\section{Results}

Cyclophosphamide decreased renal and liver functions and antioxidant enzymes, which significantly increased blood urea nitrogen- creatinine (BUN- $\mathrm{Cr}$ ), liver enzyme levels, malondialdehyde, Nuclear factor kappa $\beta$ (NF-kB) and Interleukin 1 beta (IL-1B) concentrations. Moreover, histopathological findings of the $\mathrm{CP}$ group showed that there were acute tubular necrosis and glomerular atrophy in the renal tissues and lymphocyte infiltration in the liver samples. Treatment with saponin improved hepatic and nephric functions, the pathological changes and antioxidant capacity, also, decreased lipid peroxidation and inflammation.

\section{Conclusion}

It seems that saponin could exert a hepato-nephropretective effect against cyclophosphamide toxicity.

\section{Introduction}

The kidney and liver are systems affected by chemical toxicity and chemotherapy drugs like cyclophosphamide (CP). The liver is one of the organs where drugs and chemical materials are metabolized then excreted. Liver cells or hepatocytes are to exposure with divers chemical agents maybe lead to hepatotoxicity, hepatic dysfunction and failure (Ribeiro, Yang, Patel, Madabushi, \& Strauss, 2019). The kidney has various functions such as the regulation of water and electrolyte balance, arterial pressure, body fluid osmolarity and acid-base balance. Furthermore, the kidney excretes the metabolic waste products and exogenous chemicals and the active form of vitamin $D$ is produced in the kidney by mitochondria of the proximal tubules. Also, the secretion and metabolism of hormones including erythropoietin and 1, 25(OH) 2D3 are associated to the kidneys. There are close relation between the liver 
and kidney, which this functional interaction is pivotal to maintain internal homeostasis (Brown, Bouley, Păunescu, Breton, \& Lu, 2012). Toxicity effects of chemotherapeutic agents which is widely administrated in the treatment of various types of cancers to the kidney, liver and other organs able to disrupt the physiologic functions them (Lee et al., 2008) .

These two systems (liver and kidney) metabolize and excrete chemical agents mainly involve in the material detoxification more than other organs. As a result, the toxicity of chemotherapeutic drugs can result in degenerative alterations and life-threatening kidney and liver injury (Un et al., 2020). Cyclophosphamide is a chemotherapeutic drug, is administrated in cancer diseases, it has several side effects including genital disorders, testicular damage, cardio-renal toxicity and hepatotoxicity. CP with antineoplastic properties is associated with histological and biochemical changes in the different tissues. It is known that CP interrupts the balance of free radicals and antioxidant defenses, which produced reactive oxygen species (ROS) are responsible for DNA fragmentation, necrosis, apoptosis and cell death (Cengiz et al., 2020). Oxidative injury of the DNA plays an important role in the apoptosis of the germ cells and tissues. Despite of clinical applications of CP, it has adverse influence on the liver that decomposes $\mathrm{CP}$ to active metabolites, on the kidney that excretes it. The previous studies have shown that impaired humoral secretion, infertility, DNA damage, lipid peroxidation and morphological defects in the tissues are associated to the treatment with the CP (Patwa \& Khan, 2020).

Therefore, the protection of organs such as the kidney, liver, cardiovascular system and etc against cyclophosphamide toxicity is inevitable. Herbal medicines have been confirm to affect in the inhibition of CP-induced tissue toxicity (El-Sebaey \& Abdelhamid, 2019). Saponins (SP) are natural compounds of Ginseng, Fig and Olive which they have various biological activities including anti-inflammatory, antioxidant, anti-microbial, anti-tumor and immune-modulating properties (Shao, Wang, Zhu, \& Dang, 2020; Zhao, Xue, \& Zhang, 2018). Additionally, saponins improve antioxidant capacity and normalize AST (aspartate transaminase), ALT (alanine transaminase) and ALP (alkaline phosphatase) in the liver, scavenge ROS and reduce lipid peroxidation. Also, saponins decrease heat stress and suppress oxidative stress in tubular and glomerular epithelial cells in the kidney in a dose dependent manner. It has found that some saponins obtained from plants decrease tumor growth, have preventive effect on proteinuria, therapeutic effect on liver and renal function and protective effect against cardiomyopathy (Du, Wang, Qian, Zhang, \& Chai, 2016; Zhou et al., 2016).

In recent decades, herbal remedies were evaluated to inhibit the toxicity of chemotherapeutic drugs, especially, to avoid the side effects caused by the chemical medicines. In the present study, we investigated the protective effects of saponin against CP-induced nephrotoxicity and hepatotoxicity using evaluation of the antioxidant capacity and inflammatory mediators.

\section{Method And Material}

For the study, 24 adult male NMRI mice ( 6 to 8 weeks old, weighing 20 to $25 \mathrm{~g}$ ) were divided into 4 groups and were treated for 35 days. The mice had free access to standard feed and water during the study 
period. The conditions of the animal house were under controlled temperature about $21 \pm 2^{\circ} \mathrm{C}$ and under 12-12 hour light- dark cycle with humidity (60 $\pm 5 \%)$. Principles of laboratory animals ethics with regard to international standards and the ethics committee of Ardabil University of Medical Sciences were observed in all stages of research (ethics code: IR.ARUMS.AEC.1400.019).

\section{Experimental design}

Male mice were randomly divided into 4 groups $(\mathrm{N}=6)$ with a treatment period of 35 days. The groups were as follow:

1. Control + Saline $(0.2 \mathrm{ml} /$ day, IP)

2. CP group $(15 \mathrm{mg} / \mathrm{kg} /$ week, IP)

3. SP group $(2.5 \mathrm{mg} / \mathrm{kg} / \mathrm{day}, \mathrm{IP})$

4. $\mathrm{CP}+\mathrm{SP}$ group, received $\mathrm{CP}(15 \mathrm{mg} / \mathrm{kg} /$ week $)$ with $\mathrm{SP}(2.5 \mathrm{mg} / \mathrm{kg} /$ day $)$

Cyclophosphamide (500 mg vial sigma-USA) was dissolved in normal saline in the darkness. CP was injected weekly intraperitoneally $(15 \mathrm{mg} / \mathrm{kg}$ ) (Mehraban, Gaffari Novin, Golmohammadi, \& Nazarian, 2021). Saponin powder was obtained from the company (Sigma- Aldrich- USA), and dissolved in normal saline at room temperature, away from light and under the hood. Initially, a pilot study was performed to evaluate the dosage of saponin and effective dose was $2.5 \mathrm{mg} / \mathrm{kg} /$ day (Timar \& Banaei, 2021).

Biochemical measurement

Under anesthesia, Blood samples, liver, and kidney were collected at the end of the treatment period in each group. The blood samples were centrifuged at approximately $4000 \mathrm{rmp}$ for $10 \mathrm{~min}$. The blood urea nitrogen (BUN) and creatinine ( $\mathrm{Cr}$ ) levels in the serum were determined to assess the renal function, using the Auto-analyser with original kits. The activities of ALP, ALT and AST were assayed using Randox assay kits.

Histological evaluation

Liver and renal tissue samples were fixed in $10 \%$ buffered formalin solution, dehydrated in ascending grades of alcohol and embedded in paraffin. Sections of $5 \mu \mathrm{m}$ were taken, stained with hematoxylin- eosin $(\mathrm{H} \& \mathrm{E})$, and then examined under light microscope, in a blinded manner by pathologist. The histological score was semi-quantitatively evaluated using a scale ranging from 0 to 3 ( 0 : none, 1: mild, 2: moderate, and 3: severe damage) (Golmohammadi \& Banaei, 2021).

Tissue Malondialdehyde (MDA) and Total antioxidant capacity (TAC) measurement

The liver and renal tissues were homogenized in $1.15 \% \mathrm{KCl}$. The tissue levels of MDA were assessed with the ZellBio kit (Germany). The TAC levels were determined using Radox total antioxidant status kit based on the companies method. It is the production of cation $\mathrm{ABTS}^{+}$and next to its inhibition via antioxidant compounds of the specimen (Ahmadiasl, Banaei, Alihemmati, Baradaran, \& Azimian, 2014). 
Tissue Glutathione peroxidase (GPx) and Superoxide dismutase (SOD) assessment

To measure cytosolic enzyme activity, the liver and renal samples were homogenized in $1.15 \% \mathrm{KCl}$ solution. SOD and GPx activity were measured according to the ZellBio kit (Germany) and the manufacturer protocol (Ahmadiasl, Banaei, Alihemati, Baradaran, \& Azimian, 2014).

Assessment of tissue levels of Nuclear factor kappa $\beta$ (NF-kB) and Interleukin 1 beta (IL-1B)

The liver and renal samples were collected after the treatment period and the tissue levels of NF-kB and IL-1B were measured using enzyme- linked immunosorbent assay (ELISA) kits in accordance with the manufacturer's protocol.

\section{Statistical analysis}

All the data were expressed as mean \pm standard deviation, using the one-way analysis of variance (ANOVA) followed by multiple comparison procedures using post hoc complementary test, with Graph Pad Prism version 8.4.3. Statistical significance was accepted at $P<0.05$.

\section{Results}

The aim of the current study is to determine the therapeutic and protective effects of saponin against cyclophosphamide- induced nephrotoxicity and hepatotoxicity. We hypothesized that with the intervention of appropriate therapeutics, it should be possible to manage the CP damage and provide a beneficial hepato and reno-protection. Biochemical analysis results are outlined in figures 1-5. The results of histological scores are shown in figures 7,9 .

\section{Protective effect of saponin on CP- induced alterations in serum biochemical parameters}

The levels of serum AST, ALT, and ALP enzymes were significantly elevated in the CP group in comparison with the control group ( $\mathrm{P}<0.0001$, Figures 1-3). Also, the BUN- $\mathrm{Cr}$ levels were significantly higher in the CP group compared with those from the control group $(P<0.0001$, Figures 4,5$)$. Saponin and co-administration of SP together with CP significantly reduced the levels of ALP, ALT, AST and BUN-Cr in comparison to the $\mathrm{CP}$ group.

\section{Protective effect of saponin on CP- induced renal and hepatic histopathological changes}

Histological assessment of the kidneys showed that there were no morphological changes in the control group (Figure 6A) also, healthy appearance of glomerular and tubular cells were preserved in the SP group (Figure 6C). In the CP group, acute tubular necrosis (ATN) congestion, pyknotic nuclei, glomerular atrophy and dilated bowman's space were very obvious (Figure 6B). Saponin co-administration with CP resulted in noteworthy decline in histopathological alterations induced by cyclophosphamide (Figure 6D). Correspondingly, renal histological index was significantly decreased in the saponin group in comparison to the CP group $(P<0.01$, Figure 7$)$. 
Also, histological examination of the liver showed that there were no histological changes in the control group (Figure 8A). Moreover, SP-administrated groups showed normal hepatic structure (Figure 8C). In the $\mathrm{CP}$ group, the normal histological organization of the liver tissues was disrupted. There were lymphocyte infiltration, acute triaditis and neutrophil infiltration following the treatment with CP (Figure 8B).CP plus SP demonstrated the reduction of the degenerative changes in hepatocytes (Figure 8D). Also, liver histological index was significantly decreased in the SP and CP + SP groups in comparison to the CP group $(P<0.05$, Figure 9$)$.

\section{The effect of saponin and cyclophosphamide on the MDA and TAC levels}

As shown in the Table 1, in the CP group, the MDA levels were insignificantly increased as compared to the control group. Saponin administration significantly reduced MDA concentration than the CP group (P $<0.01$ ). Besides, the CP administration decreased TAC levels in contrast to the control group. But, saponin treatment significantly elevated TAC levels in comparison with the CP group $(P<0.05)$.

\section{The effect of saponin and cyclophosphamide on the SOD and GPX activity}

The mean activity of GPx and SOD in the liver and kidney in the CP group insignificantly decreased in comparison to the control group. The saponin treated groups showed a considerable increase of SOD and GPx levels as compared to the CP group (Table 1).

\section{The effect of saponin and cyclophosphamide on the NF-kB and IL-1B productions}

We assessed whether saponin decreases the production of the pro-inflammatory cytokines: NF-kB and IL$1 \mathrm{~B}$ induced by cyclophosphamide. The toxic effects of $\mathrm{CP}$ resulted in significant elevation of NF-kB and IL-1B concentrations in kidney and liver tissues when compared to the control group $(P<0.001)$. However, the tissue levels of these cytokines were significantly reduced in the saponin $(P<0.001)$ and CP plus SP $(P<0.05)$ groups in comparison to the $C P$ group (Figures 10 and 11). These results indicate that saponin alone and co-administration of CP with SP decrease the NF-kB and IL-1B productions.

\section{Discussion}

The liver and kidney are systems affected by chemotherapy drugs like cyclophosphamide. Acute and chronic exposure to some chemical agents result in nephrotoxicity and hepatotoxicity (Bhat, Kalthur, Padmashali, \& Monappa, 2018). Inflammation is characterized by the protective response of the immune system against exogenous chemicals are metabolized and finally excreted by liver and kidney. In this process, pro-inflammatory cytokines including tumor necrosis factor alpha (TNF-a), interleukins and mediators such as nitric oxide (NO) increase in the body and reactive oxygen species (ROS) are produced. Generation of ROS, including the hydroxyl radical $(\mathrm{OH})$, hydrogen peroxide and superoxide anions or the reduction of antioxidant defense leads to oxidative stress, which plays a critical role in the development of liver and renal injury and even organ failure. Therefore, ROS scavengers and antioxidants that remove free radicals, moreover, anti-inflammatory herbal medicines (natural remedies) can protect against renal 
and liver dysfunction mediated by cyclophosphamide (Kocahan, Dogan, Erdemli, \& Taskin, 2017; Zhai et al., 2018).

Many investigations have been done about the antioxidant, anti-inflammatory and immune-regulator role of saponins. Saponins reduce the produced free radicals, regulate the expression of antioxidant enzymes and eliminate the toxic effects of the industrial chemicals that lead to resolution of inflammation and return to hemostasis (Ma, Jiang, Yuan, Qiu, \& Zhu, 2019; Yao et al., 2019). Therefore, in the present study, we hypothesized saponins with antioxidant and anti-inflammatory capacity able to inhibit liver and kidney toxicity due to the treatment with cyclophosphamide.

Our result in the current study indicated that exposure with CP significantly increased the serum levels of ALP, ALT, AST and BUN- Cr, suggesting a liver and renal dysfunction which was greatly decreased after saponin treatment. Saponin and co-administration of SP together with CP significantly reduced these parameters. Probably, antioxidant properties of saponin could be effective in the improvement of hepatic and renal function. On the other hand, histological evaluation showed that CP caused glomerular and tubular changes, as shown by glomerular atrophy, dilated bowman's space, acute tubular necrosis and congestion. Saponin and CP plus SP attenuated the pathological changes in renal tissue caused by cyclophosphamide. Also, renal histological index was decreased by the saponin treatment. This cytoprotective effect of saponin may be due to its powerful antioxidant capacity (Hu et al., 2019).

In addition, cyclophosphamide resulted in the pathological changes in the hepatocytes, including acute triaditis and lymphocyte infiltration. As the administration of saponin and CP plus SP reduced the morphological changes of the liver tissues. Correspondingly, liver histological index was significantly decreased with the saponin treatment in the effective dose of $2.5 \mathrm{mg} / \mathrm{kg}$. Probably, these protective effects of saponin may be due to potent anti-inflammatory and antioxidant properties (Wang, Xiang, Yi, \& $\mathrm{He}, 2017)$, which were documented by the recovery and regeneration of pathological defects in the liver and renal structure.

The lipid peroxidation, as a free radical generating system, has been reported to be closely related to CPinduced tissue damages, and MDA is a good marker of the degree of lipid peroxidation (Mehraban et al., 2021). In the present experiment, the MDA levels were elevated and the activity of GPx, TAC and SOD were reduced by $\mathrm{CP}$ treatment. These data are consistent with previously published findings about the effects of CP on the organ toxicity (Duggina, Kalla, Varikasuvu, Bukke, \& Tartte, 2015). The administration of CP led to oxidative stress, lipid peroxidation in renal and hepatic tissues and reduced antioxidant defenses. Increased oxidative stress is an important participant in the induced toxicity by CP. Therefore, compounds that can inhibit the production of free radicals will be of particular importance. Saponin has a more significant antioxidant effect, which our results confirmed that it decreased the amount of oxidative stress and subsequently lipid peroxidation and improved the antioxidant capacity. Thus documenting the ability of saponin's dual function as both an enhancer of antioxidative enzyme defenses and a free radical scavenger. 
Exposure to chemicals and drugs result in inflammation that is characterized by the protective response of the immune system against these agents. Pro-inflammatory cytokines including tumor necrosis factor alpha (TNF-a), IL-1B and IL-6 increase in the body. Transcription of these interleukins are induced by an important protein in inflammatory process, NF-kB (Ginwala, Bhavsar, Chigbu, Jain, \& Khan, 2019). Oxidative stress has been considered to be related with NF-kB. Actually, a vicious cycle has been suggested between the oxidative stress and NF-kB signaling pathway. Oxidative stress is crucial for the activation of the NF-kB cascade. On the other hand, the expression of NF-kB leads to the aggravation of free radical generation (Morgan \& Liu, 2011).

Our results indicated that $\mathrm{CP}$ created a state of oxidative stress in kidney and liver tissues and then induced the production of pro-inflammatory cytokines such as NF-kB and IL-1B from these two organs which these findings are consistent with previous researches in this regard (Jnaneshwari et al., 2014). Usually, inhibition of inflammation is the most main actions in controlling the toxicity effect of these agents. The inflammatory conditions have been treated with the natural compounds. To prevent unwanted side effects of anti-inflammatory medicines, nonsteroidal anti-inflammatory drugs (NSAIDs), it is necessary to find out alternative anti-inflammatory agents with more efficacy than NSAIDs but with fewer side effects. Our findings demonstrated that saponin significantly could inhibit the production of inflammatory mediators induced by CP. Also, CP plus SP significantly reduced the NF-kB and IL-1B in kidney and liver tissues that were increased by CP. Probably, the high antioxidant and anti-inflammatory properties of saponin is responsible for ROS detoxification and inflammatory reduction.

In a nutshell, diagram 1 illustrates saponins-mediated hepato and renoprotection by the inhibition of oxidative stress.

\section{Conclusion}

In conclusion, liver and renal dysfunction are the most important complications of chemotherapy drugs such as cyclophosphamide. Increased oxidative stress and inflammation is an accepted participant in the induced toxicity by CP. Saponins have significant antioxidant and anti-inflammatory actions and are wellknown free radical scavengers. Our findings showed that the administration of saponin or CP plus saponin appears to exert a protective role on CP-induced hepatic and nephric injury, as shown by the reduction of liver and renal dysfunction, oxidative stress, production of pro-inflammatory mediators and pathological changes and improvement of antioxidant capacity. However, further investigations is necessary to clarify the exact mechanisms mediating the therapeutic properties of saponin against cyclophosphamide toxicity.

\section{Declarations}

\section{Funding}

This study was funded by Ardabil University of Medical Sciences. 


\section{Ethical Approval}

This study was approved by Ardabil University of Medical Sciences (Ethics code:

IR.ARUMS.AEC.1400.019).

\section{Authors contributions}

MG.G. and Sh. B. conceived and designed research. Sh. B. did histological evaluation, statistical analysis and manuscript writing. All authors read and approved the final manuscript. The authors declare that all data were generated in-house and that no paper mill was used.

\section{Consent to participate}

Not applicable.

\section{Consent to publish}

Not applicable.

\section{Competing interest}

The authors declare no competing interests.

\section{Data Availability}

The data that support the finding of this study are available through the following link (Supplementary files).

\section{References}

1. Ahmadiasl, N., Banaei, S., Alihemati, A., Baradaran, B., \& Azimian, E. (2014). Effect of a combined treatment with erythropoietin and melatonin on renal ischemia reperfusion injury in male rats. Clin Exp Nephrol, 18(6), 855-864. doi: 10.1007/s10157-014-0937-6

2. Ahmadiasl, N., Banaei, S., Alihemmati, A., Baradaran, B., \& Azimian, E. (2014). The anti-inflammatory effect of erythropoietin and melatonin on renal ischemia reperfusion injury in male rats. Adv Pharm Bull, 4(1), 49-54. doi: 10.5681/apb.2014.008

3. Bhat, N., Kalthur, S. G., Padmashali, S., \& Monappa, V. (2018). Toxic Effects of Different Doses of Cyclophosphamide on Liver and Kidney Tissue in Swiss Albino Mice: A Histopathological Study. Ethiop J Health Sci, 28(6), 711-716. doi: 10.4314/ejhs.v28i6.5

4. Brown, D., Bouley, R., Păunescu, T. G., Breton, S., \& Lu, H. A. (2012). New insights into the dynamic regulation of water and acid-base balance by renal epithelial cells. Am J Physiol Cell Physiol, 302(10), C1421-1433. doi: 10.1152/ajpcell.00085.2012 
5. Cengiz, M., Sahinturk, V., Yildiz, S. C., Şahin İ, K., Bilici, N., Yaman, S. O., .. Ayhanci, A. (2020). Cyclophosphamide induced oxidative stress, lipid per oxidation, apoptosis and histopathological changes in rats: Protective role of boron. J Trace Elem Med Biol, 62, 126574. doi: 10.1016/j.jtemb.2020.126574

6. Du, Y. G., Wang, L. P., Qian, J. W., Zhang, K. N., \& Chai, K. F. (2016). Panax notoginseng saponins protect kidney from diabetes by up-regulating silent information regulator 1 and activating antioxidant proteins in rats. Chin J Integr Med, 22(12), 910-917. doi: 10.1007/s11655-015-2446-1

7. Duggina, P., Kalla, C. M., Varikasuvu, S. R., Bukke, S., \& Tartte, V. (2015). Protective effect of centella triterpene saponins against cyclophosphamide-induced immune and hepatic system dysfunction in rats: its possible mechanisms of action. J Physiol Biochem, 71(3), 435-454. doi: 10.1007/s13105015-0423-y

8. El-Sebaey, A. M., \& Abdelhamid, F. M. (2019). Protective effects of garlic extract against hematological alterations, immunosuppression, hepatic oxidative stress, and renal damage induced by cyclophosphamide in rats. 26(15), 15559-15572. doi: 10.1007/s11356-019-04993-7

9. Ginwala, R., Bhavsar, R., Chigbu, D. I., Jain, P., \& Khan, Z. K. (2019). Potential Role of Flavonoids in Treating Chronic Inflammatory Diseases with a Special Focus on the Anti-Inflammatory Activity of Apigenin. Antioxidants (Basel), 8(2). doi: 10.3390/antiox8020035

10. Golmohammadi, M. G., \& Banaei, S. (2021). Mechanistic Evaluation of Linalool Effect against Renal Ischemia- Reperfusion Injury in Rats. doi: 10.1055/a-1488-5904

11. Hu, S., Liu, T., Wu, Y., Yang, W., Hu, S., Sun, Z., . . Du, S. (2019). Panax notoginseng saponins suppress lipopolysaccharide-induced barrier disruption and monocyte adhesion on bEnd. 3 cells via the opposite modulation of Nrf2 antioxidant and NF-KB inflammatory pathways. Phytother Res, 33(12), 3163-3176. doi: 10.1002/ptr.6488

12. Jnaneshwari, S., Hemshekhar, M., Thushara, R. M., Sundaram, M. S., Santhosh, M. S., Sunitha, K., . . Girish, K. S. (2014). Sesamol ameliorates cyclophosphamide-induced hepatotoxicity by modulating oxidative stress and inflammatory mediators. Anticancer Agents Med Chem, 14(7), 975-983. doi: $10.2174 / 1871520613666131224123346$

13. Kocahan, S., Dogan, Z., Erdemli, E., \& Taskin, E. (2017). Protective Effect of Quercetin Against Oxidative Stress-induced Toxicity Associated With Doxorubicin and Cyclophosphamide in Rat Kidney and Liver Tissue. Iran J Kidney Dis, 11(2), 124-131.

14. Lee, C. K., Son, S. H., Park, K. K., Park, J. H., Lim, S. S., \& Chung, W. Y. (2008). Isoliquiritigenin inhibits tumor growth and protects the kidney and liver against chemotherapy-induced toxicity in a mouse xenograft model of colon carcinoma. J Pharmacol Sci, 106(3), 444-451. doi:

10.1254/jphs.fp0071498

15. Ma, Q., Jiang, J. G., Yuan, X., Qiu, K., \& Zhu, W. (2019). Comparative antitumor and anti-inflammatory effects of flavonoids, saponins, polysaccharides, essential oil, coumarin and alkaloids from Cirsium japonicum DC. Food Chem Toxicol, 125, 422-429. doi: 10.1016/j.fct.2019.01.020 
16. Mehraban, Z., Gaffari Novin, M., Golmohammadi, M. G., \& Nazarian, H. (2021). Effect of Ceratonia siliqua L. extract on DNA Fragmentation of Sperm in Adult Male Mice Treated with Cyclophosphamide. Reprod Sci, 28(4), 974-981. doi: 10.1007/s43032-020-00322-3

17. Morgan, M. J., \& Liu, Z. G. (2011). Crosstalk of reactive oxygen species and NF-kB signaling. Cell Res, 21(1), 103-115. doi: 10.1038/cr.2010.178

18. Patwa, J., \& Khan, S. (2020). Nicotinamide attenuates cyclophosphamide-induced hepatotoxicity in SD rats by reducing oxidative stress and apoptosis. 34(10), e22558. doi: 10.1002/jbt.22558

19. Ribeiro, A. J. S., Yang, X., Patel, V., Madabushi, R., \& Strauss, D. G. (2019). Liver Microphysiological Systems for Predicting and Evaluating Drug Effects. Clin Pharmacol Ther, 106(1), 139-147. doi: 10.1002/cpt.1458

20. Shao, X., Wang, X., Zhu, K., \& Dang, Y. (2020). Synthesis of Sea Cucumber Saponins with Antitumor Activities. 85(19), 12080-12096. doi: 10.1021/acs.joc.0c01191

21. Timar, M., \& Banaei, S. (2021). Protective effect of saponin on sperm DNA fragmentation of mice treated with cyclophosphamide. e14336. doi: 10.1111/and.14336

22. Un, H., Ugan, R. A., Kose, D., Bayir, Y., Cadirci, E., Selli, J., \& Halici, Z. (2020). A novel effect of Aprepitant: Protection for cisplatin-induced nephrotoxicity and hepatotoxicity. Eur J Pharmacol, 880, 173168. doi: 10.1016/j.ejphar.2020.173168

23. Wang, Y., Xiang, L., Yi, X., \& He, X. (2017). Potential Anti-inflammatory Steroidal Saponins from the Berries of Solanum nigrum L. (European Black Nightshade). 65(21), 4262-4272. doi: 10.1021/acs.jafc.7b00985

24. Yao, F., Xue, Q., Li, K., Cao, X., Sun, L., \& Liu, Y. (2019). Phenolic Compounds and Ginsenosides in Ginseng Shoots and Their Antioxidant and Anti-Inflammatory Capacities in LPS-Induced RAW264.7 Mouse Macrophages. 20(12). doi: 10.3390/ijms20122951

25. Zhai, J., Zhang, F., Gao, S., Chen, L., Feng, G., Yin, J., \& Chen, W. (2018). Schisandra chinensis extract decreases chloroacetaldehyde production in rats and attenuates cyclophosphamide toxicity in liver, kidney and brain. J Ethnopharmacol, 210, 223-231. doi: 10.1016/j.jep.2017.08.020

26. Zhao, Y. C., Xue, C. H., \& Zhang, T. T. (2018). Saponins from Sea Cucumber and Their Biological Activities. 66(28), 7222-7237. doi: 10.1021/acs.jafc.8b01770

27. Zhou, Y., Tong, X., Ren, S., Wang, X., Chen, J., Mu, Y., . . Liu, P. (2016). Synergistic anti-liver fibrosis actions of total astragalus saponins and glycyrrhizic acid via TGF- $\beta 1 /$ Smads signaling pathway modulation. J Ethnopharmacol, 190, 83-90. doi: 10.1016/j.jep.2016.06.011

\section{Tables}


Table 1

Protective effect of saponin on cyclophosphamide- induced changes in renal and hepatic TAC, MDA and antioxidant enzyme levels.

\begin{tabular}{|c|c|c|c|c|}
\hline Groups & Control & $\mathrm{CP}$ & SP & $\mathrm{CP}+\mathrm{SP}$ \\
\hline \multicolumn{5}{|l|}{ Kidney } \\
\hline MDA (nmol/mg protein) & $1.61 \pm 0.44$ & $1.89 \pm 0.22$ & $1.15 \pm 0.14 \star \star$ & $0.88 \pm 0.10 \star \star \star *$ \\
\hline TAC (mmol/liter) & $0.51 \pm 0.07$ & $0.43 \pm 0.11$ & $0.63 \pm 0.03^{\star \star}$ & $0.62 \pm 0.06^{*}$ \\
\hline SOD (U/mg protein) & $8.87 \pm 1.82$ & $7.43 \pm 1.02$ & $8.53 \pm 1.67$ & $8.38 \pm 1.02$ \\
\hline GPx (U/mg protein) & $5.18 \pm 1.33$ & $4.39 \pm 0.38$ & $5.12 \pm 0.83$ & $4.70 \pm 0.91$ \\
\hline \multicolumn{5}{|l|}{ Liver } \\
\hline MDA (nmol/mg protein) & $0.67 \pm 0.15$ & $0.82 \pm 0.21$ & $0.68 \pm 0.18$ & $0.48 \pm 0.06^{*}$ \\
\hline TAC (mmol/liter) & $0.77 \pm 0.07$ & $0.70 \pm 0.19$ & $0.92 \pm 0.16$ & $1.01 \pm 0.14^{\star}$ \\
\hline SOD (U/mg protein) & $9.47 \pm 1.16$ & $8.23 \pm 0.82$ & $10.11 \pm 0.56$ & $8.63 \pm 1.39$ \\
\hline GPx (U/mg protein) & $6.26 \pm 0.57$ & $4.93 \pm 1.00$ & $6.06 \pm 0.48$ & $5.70 \pm 0.77$ \\
\hline \multicolumn{5}{|c|}{ The results were expressed as mean \pm standard deviation. } \\
\hline \multicolumn{5}{|c|}{ 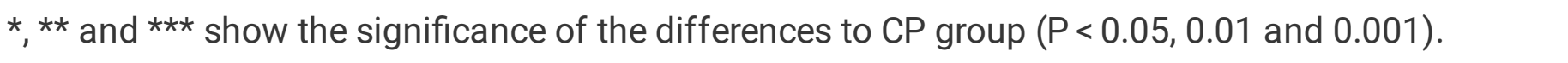 } \\
\hline \multicolumn{5}{|c|}{$\begin{array}{l}\text { SP; saponin, CP; cyclophosphamide, MDA; malondialdehyde, TAC; total antioxidant capacity, SOD; } \\
\text { superoxide dismutase, GPx; glutathione peroxidase. }\end{array}$} \\
\hline
\end{tabular}

\section{Figures}

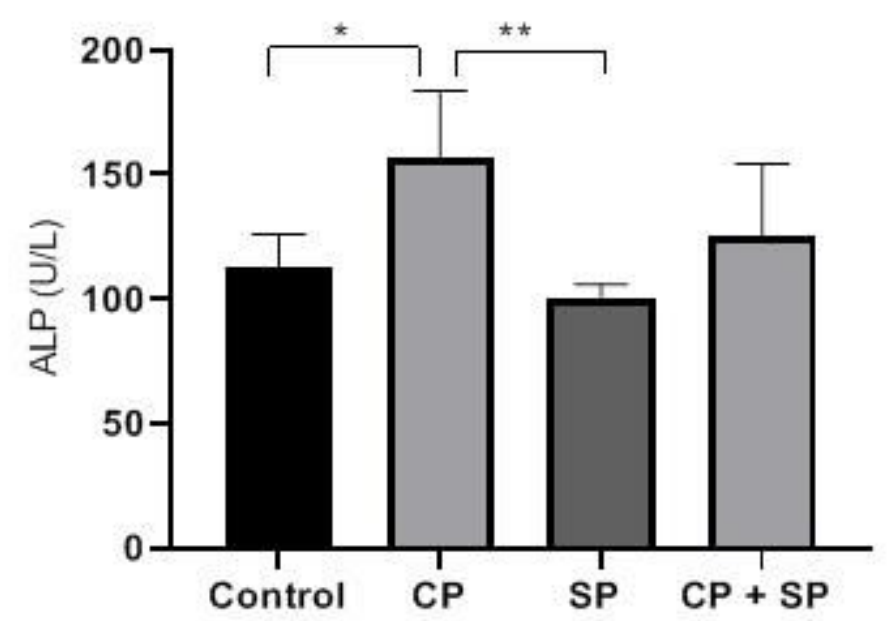

Figure 1 
The effect of cyclophosphamide (CP) and saponin (SP) on serum levels of ALP (alkaline phosphatase) in treated mice. ${ }^{*} P<0.05$ versus control group. ${ }^{* \star} P<0.01$ versus $C P$ group.

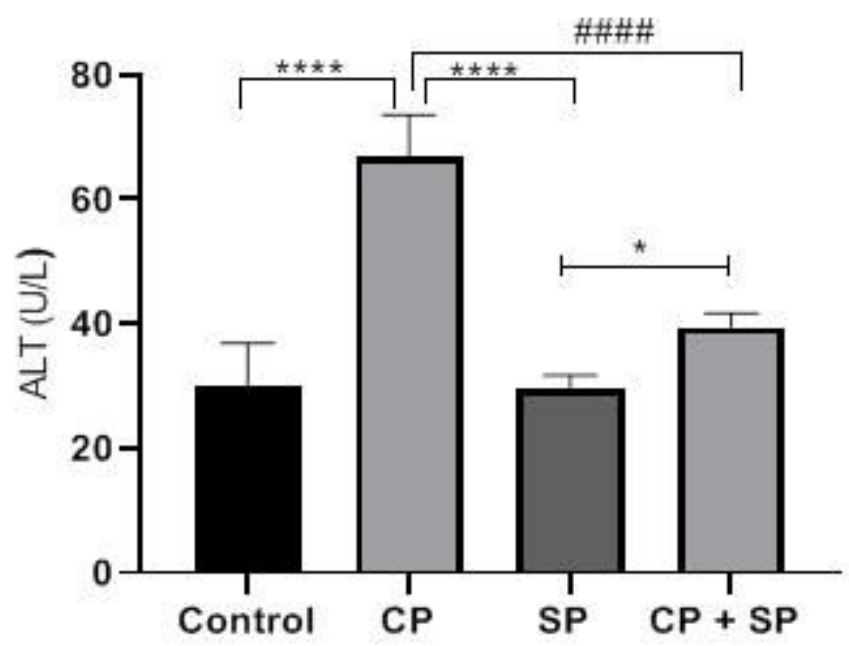

Figure 2

Alanine transaminase (ALT) levels. ${ }^{\star \star \star *} \mathrm{P}<0.0001$ versus control group. ${ }^{\# \# \# P<0.0001,{ }^{\star} * \star \star * P<0.0001}$ versus $\mathrm{CP}$ group. ${ }^{*} \mathrm{P}<0.05$ versus $\mathrm{SP}$ group.

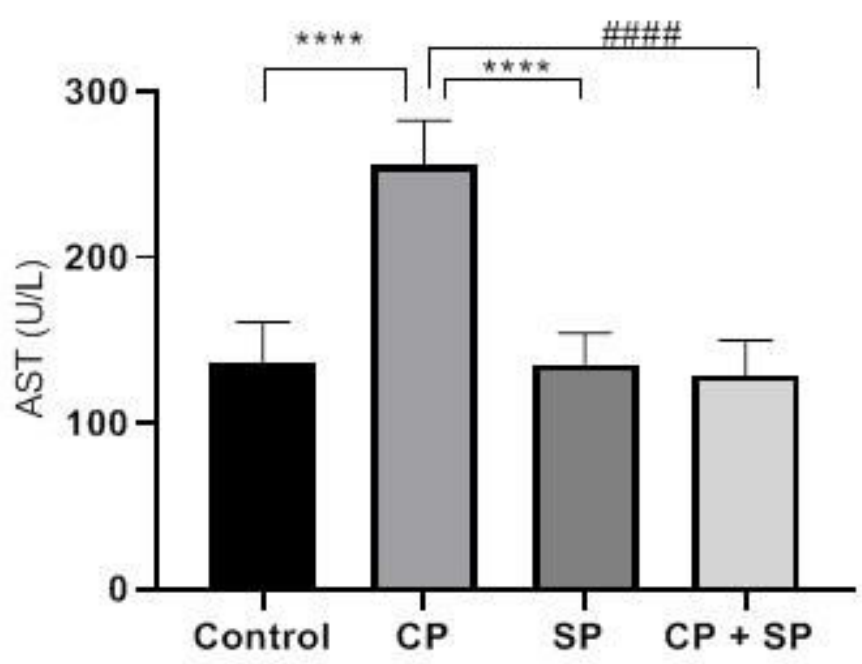

Figure 3

Aspartate transaminase (AST) levels. ${ }^{\star \star \star \star} \mathrm{P}<0.0001$ versus control group. ${ }^{\# \# \# P<0.0001,{ }^{*} \star * \star P}<0.0001$ versus CP group. 


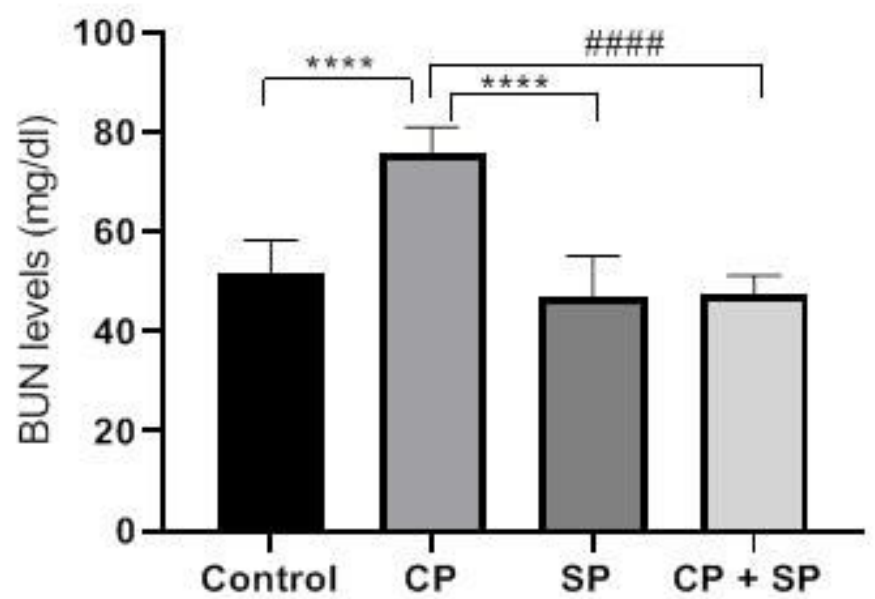

Figure 4

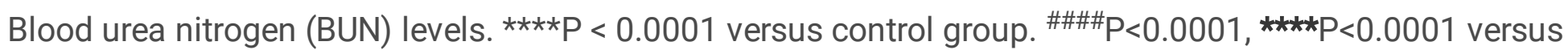
CP group.

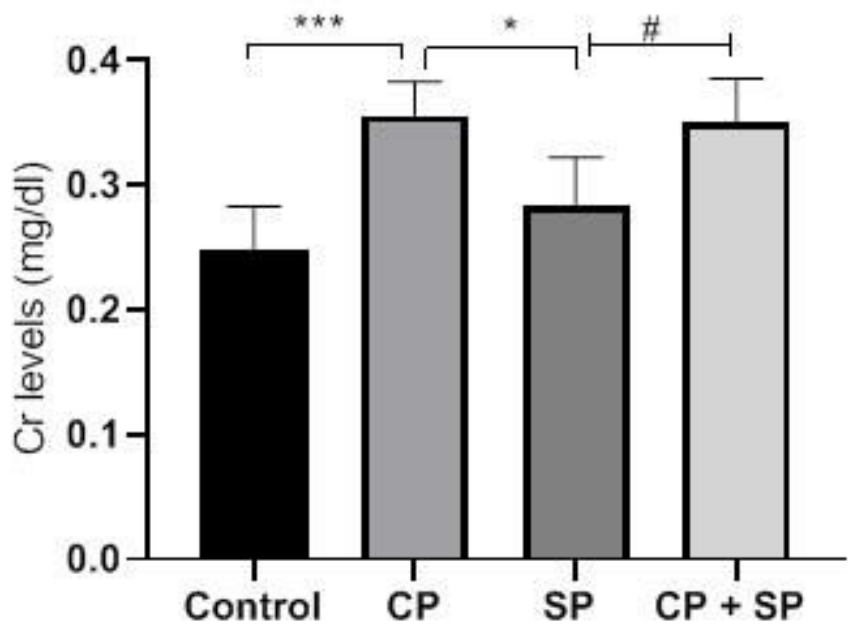

Figure 5

Creatinine $(\mathrm{Cr})$ levels. ${ }^{* \star *} \mathrm{P}<0.001$ versus control group. ${ }^{*} \mathrm{P}<0.05$ versus $\mathrm{CP}$ group. ${ }^{\#} \mathrm{P}<0.05$ versus SP group. 

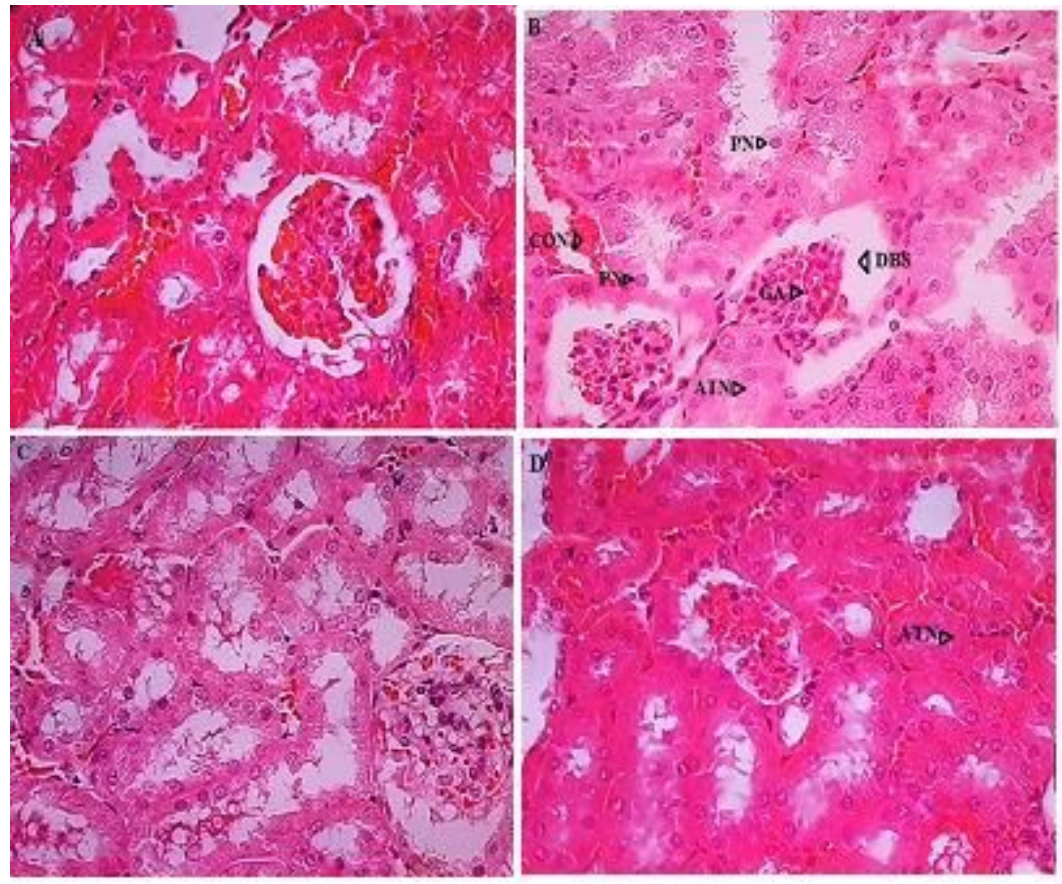

\section{Figure 6}

(A and C) Photomicrograph of the kidney of normal control and SP groups in treated mice indicates the normal renal tissue structure, healthy appearance of glomerular and tubular cells. (B) CP-administered mice had congestion (CON), pyknotic nuclei (PN), glomerular atrophy (GA), dilated bowman's space (DBS) and acute tubular necrosis (ATN). (D) Acute tubular necrosis (ATN) in CP + SP group (40 × H\&E).

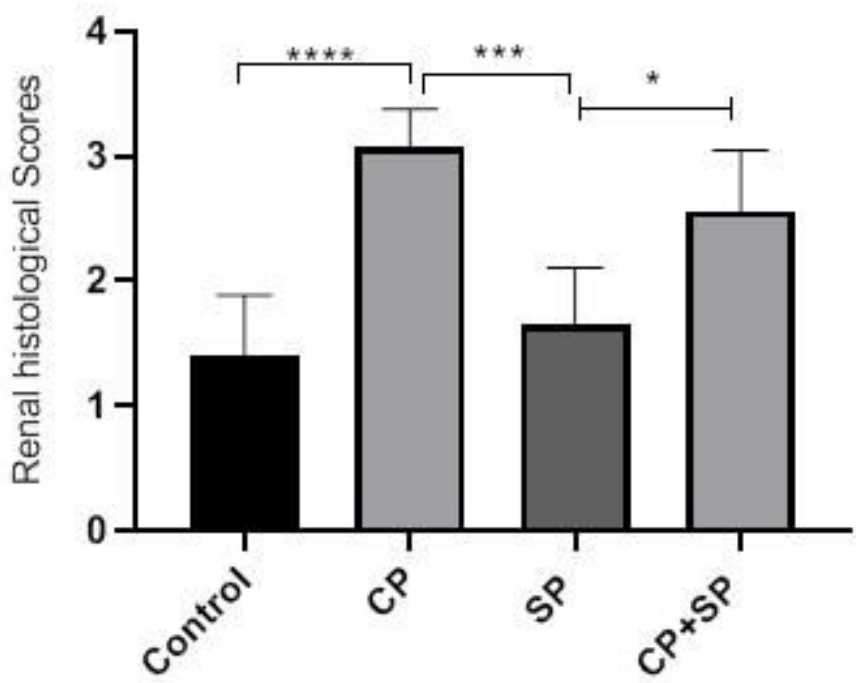

Figure 7

Semi-quantitiative analysis of protective effect of saponin against CP-induced the kidney damage. The histological score was semi-quantitatively evaluated using a scale ranging from 0 to 3 (0: none, 1: mild, 2:

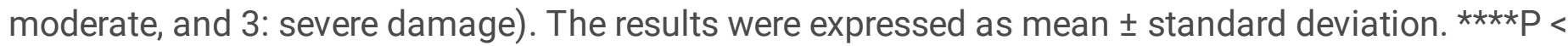

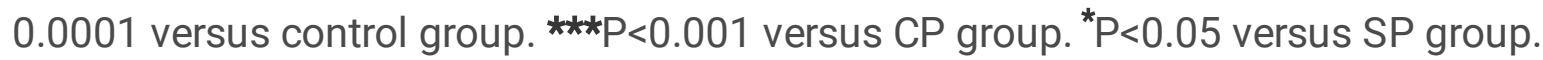




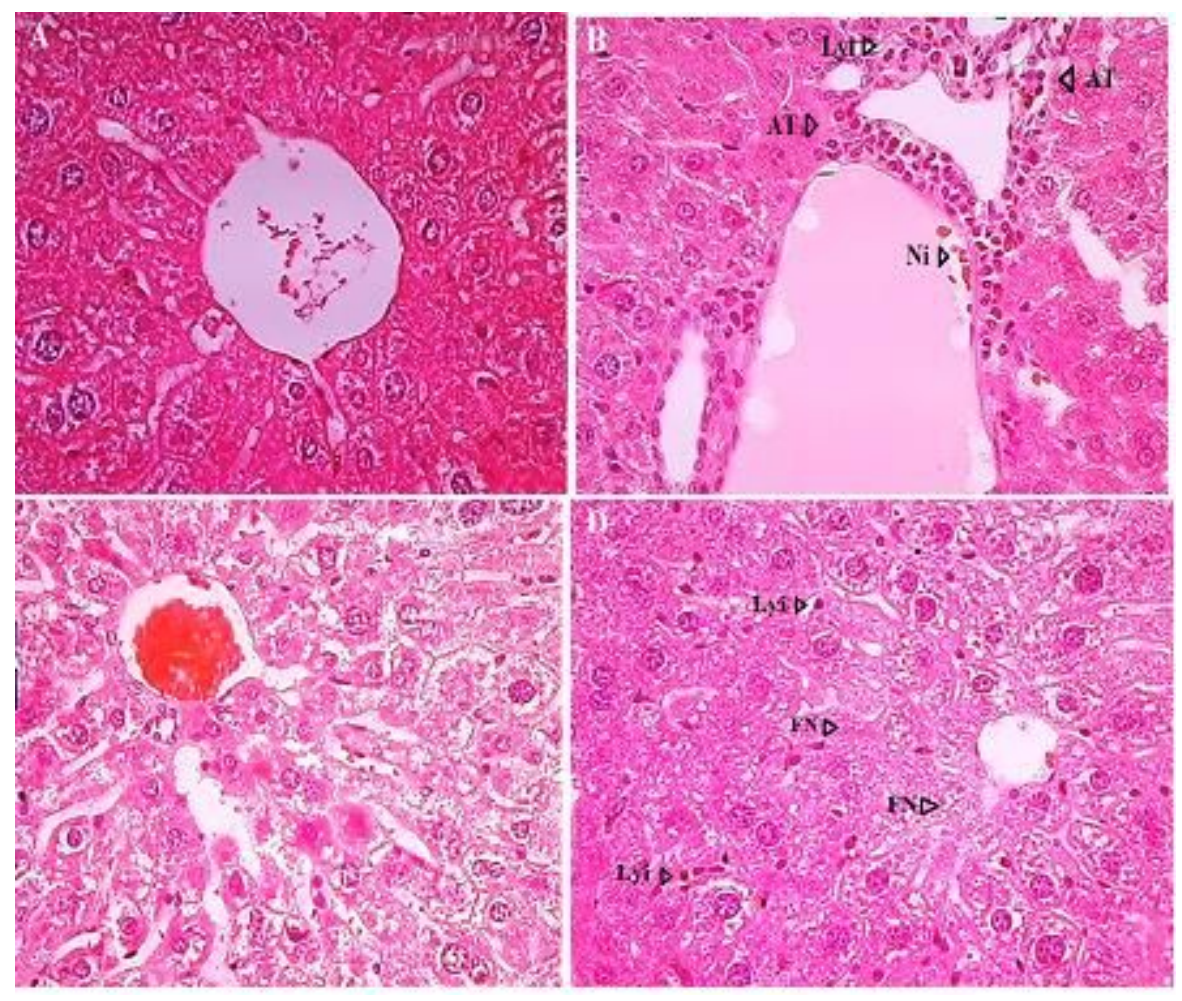

Figure 8

(A and C) Photomicrograph of the liver of normal control and SP groups in treated mice indicates the normal hepatic tissue structure. (B) CP- administered mice had lymphocyte infiltration (Lyi), acute triaditis (AT) and neutrophil infiltration (Ni). (D) Lymphocyte infiltration (Lyi) and focal necrosis (FN) in CP + SP group $(40 \times \mathrm{H} \& \mathrm{E})$.

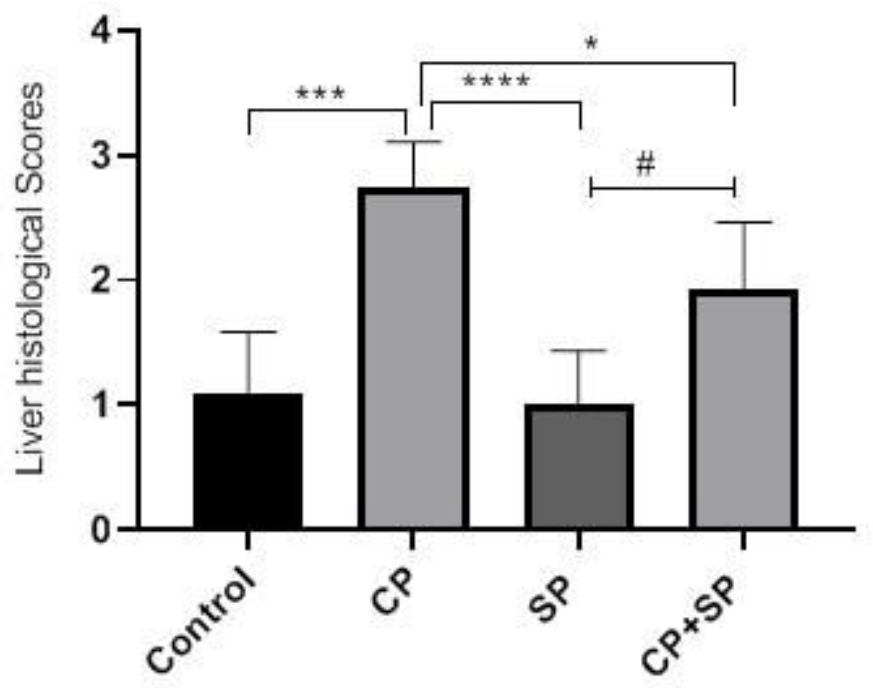

Figure 9

Semi-quantitiative analysis of protective effect of saponin against CP- induced the liver damage. The histological score was semi-quantitatively evaluated using a scale ranging from 0 to 3 (0: none, 1: mild, 2: 
moderate, and 3: severe damage). The results were expressed as mean \pm standard deviation. ${ }^{\star \star \star} \mathrm{P}<0.001$ versus control group. ${ }^{*} \mathrm{P}<0.05,{ }^{* \star \star *} \mathrm{P}<0.0001$ versus $\mathrm{CP}$ group. ${ }^{*} \mathrm{P}<0.05$ versus $\mathrm{SP}$ group.

a

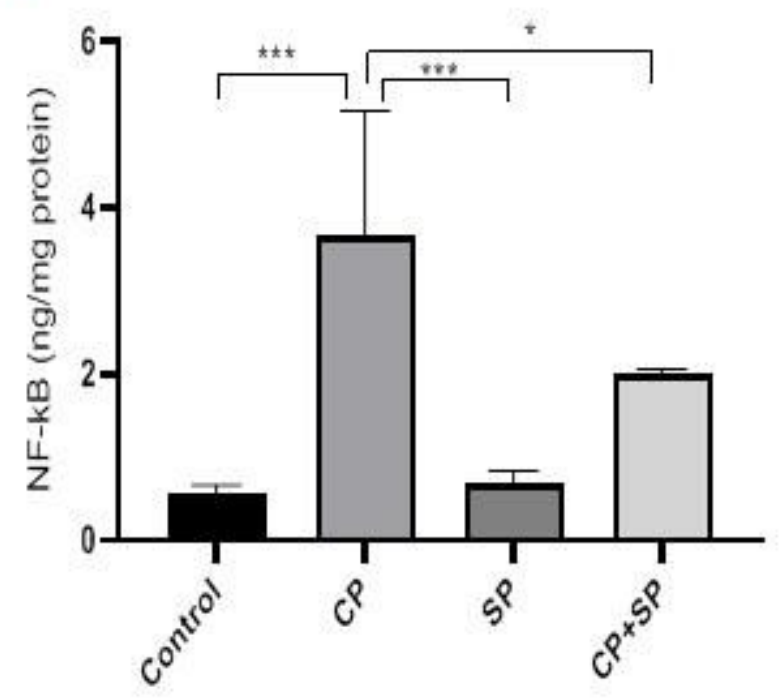

b

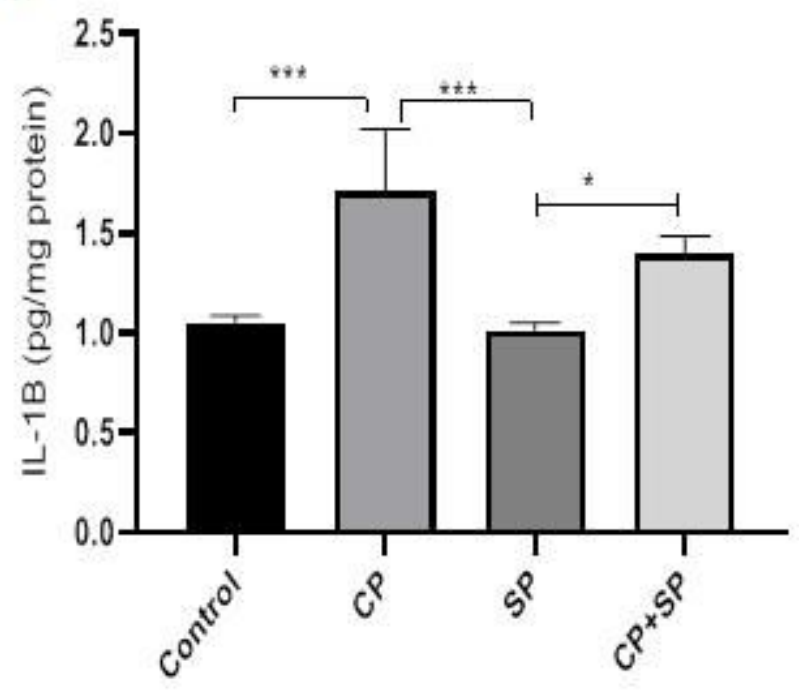

Figure 10

The effect of cyclophosphamide (CP) and saponin (SP) on NF-kB and IL-B levels in renal tissue of treated mice. ${ }^{\star \star *} \mathrm{P}<0.001$ versus control group. ${ }^{\star \star \star} \mathrm{P}<0.001,{ }^{\star} \mathrm{P}<0.05$ versus $\mathrm{CP}$ group.

a

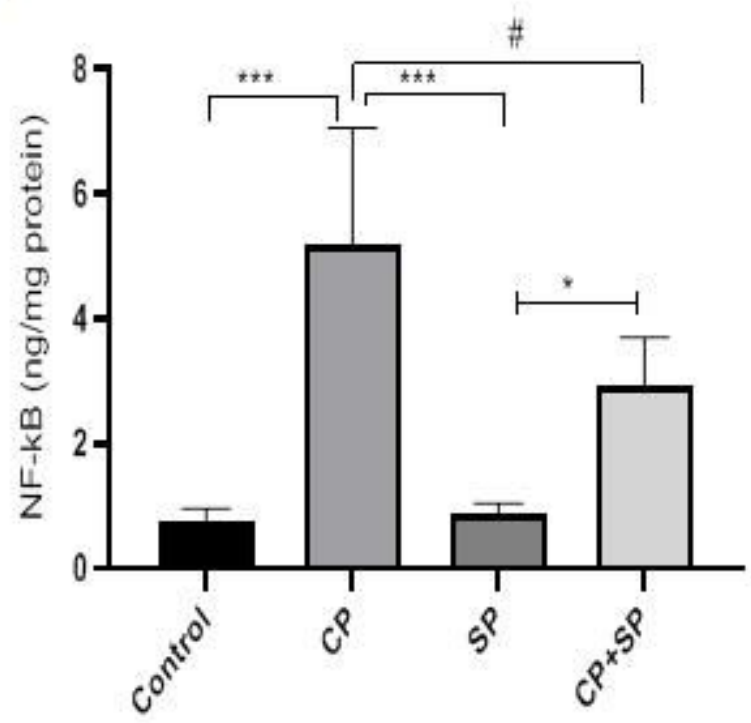

b

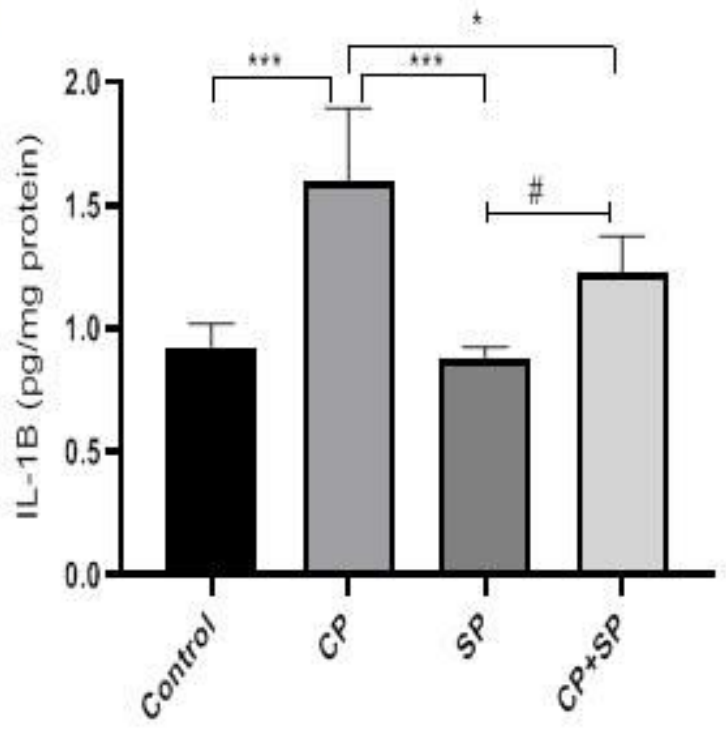

Figure 11 
NF-kB and IL-B levels in liver tissue of treated mice. ${ }^{\star \star *} \mathrm{P}<0.001$ versus control group. ${ }^{\star \star \star} \mathrm{P}<0.001$, $\star P<0.05$ versus $C P$ group.

\section{Supplementary Files}

This is a list of supplementary files associated with this preprint. Click to download.

- ALP.pzfx

- ALT.pzfx

- AST.pzfx

- BUN.pzfx

- Cr.pzfx 